\title{
Dormitory Model Based on the Arrangement of Pyroelectric Infrared Sensor
}

\author{
Yuchen Huang ${ }^{1, a}$ and Yanhao Wang ${ }^{1, a}$ \\ ${ }^{1}$ North China Electric Power University, Beijing, 102206 \\ aalanncepu@foxmail.com
}

\begin{abstract}
Keywords: Saving electricity; Mathematical modeling; Pyroelectric infrared sensor; Installation location
\end{abstract}

\begin{abstract}
Recently, how to save electricity has become the focus of social concern. In order to avoid wasting electricity, many universities limit the power supply. However, there are still many aspects need to be improved in dormitory electricity management. Installing some pyroelectric infrared sensors to detect whether someone is in the dormitory can effectively solve the problem of forgetting to turn off the lights after leaving. This paper mainly applies the mathematical modeling method to calculate the required number and the installation location of sensors. When the sensors find that no one is in the hostel we can use our system to cut all power off, so as to save the electricity.
\end{abstract}

\section{Background}

In recent years, carbon dioxide and other greenhouse gas emissions lead the global climate deterioration, and energy problem has become a major issue all around the world. The power industry which takes an essential part in energy-saving and emission-reduction should be attached enough importance. As a college student, we are supposed to focus on the university dormitory electricity situation.

Many students forget to turn off the power when they leave the dormitories, which not only shorten the working life of the electrical appliances, but also lead to a large amount of energy waste, reducing the safety of the dormitory. Therefore, we hope that the system can detect whether someone is in the dormitory via sensors, and the switch can be cut off automatically when no people in the room.

In this paper, we selected the pyroelectric infrared sensor as the detection element. Usually, only one sensor often can't meet the sensitivity requirements, so we need to determine the number of sensors and their installation location, accurately determining whether there are people in the hostel.

\section{Problem Analysis}

The induction range of a pyroelectric infrared sensor is limited, and beyond this range of areas the test result is inaccurate. Usually, this induction range can be described through induction angle and distance. When people enter the induction range, the output is a high level. When people leave the induction range, the time-delay sensor automatically change its output from a high level to a low level. There is no doubt that the large shelters in the dormitory will affect the sensor's sensing area. So as to ensure that people can be sensed by the sensor no matter what place they stand in the dormitory, and taking economic problems into consideration, we should determine the minimum number of sensors. At the same time, we also need to find the best installation locations of these sensors.

\section{Dormitory Model and Assumptions}

Considering the majority of college dorms' sizes, we simplify the dormitory into a $7.2 * 4.3 * 3.3$ $\mathrm{m}^{3}$ rectangular box with three bunk beds $\left(0.9 * 2 \mathrm{~m}^{2}\right)$ and a cupboard. These three bunk beds are arranged in the three corners of the dormitory, and the remaining corner places a cupboard. 
The model assumptions are as follows:

We assume that only the bed and the cabinet will affect the sensor sensing range, ignoring the other shelters;

We assume that the sensor's induction angle is 40 degrees to 100 degrees, and its induction distance is within 7 meters.

In our model, we assume that the indoor temperature is within the sensor operating temperature range;

We neglect the light close to the lens, the flow of wind and other sources of interference on the sensor.

\section{Spatial Modeling and Initial Establishment of Scoring Function}

We can easily know the size as well as the position of the bunk beds and cupboards from the above-mentioned simplified model of the dormitory. In order to find the optimal scenario, we build up a scoring system, and the more scores the scenario gets, the better this scenario is.

First, we define the volume of a cell is $0.1 * 0.1 * 0.1 \mathrm{~m}^{3}$, and according to this, we divide the hostel into 102168 cells. In this model, each cell of the hostel is a fixed point, and the sensor coordinates are variables. Our scoring functions include the distance scoring function and the angle scoring function. The distance scoring function takes the distance as the independent variable and the score as the dependent variable. Based on the working characteristics of the sensor, we set the segmentation function. The angle scoring function takes the angle as the independent variable and the score as the dependent variable. Since the sensor sensing angle is 40 degrees to 100 degrees, the corresponding scoring function can be obtained. Then, because the light travels along the straight line, when the light illuminates the object, it will cast the shadow. Additionally, we introduce the shadow area to describe the shelter of upper berth, and if the cell is in the shadow, we think its score is 0 .

\section{The Design of Scoring Function}

The Distance Scoring Function. Take a, b, c as the cell coordinates, x1, y1, z1 as the sensor coordinates, then the scoring functions are as follows:

$\left\{\begin{array}{l}1, \tan ^{-1} \sqrt{(\mathrm{x} 1-\mathrm{a})^{2}+(\mathrm{y} 1-\mathrm{b})^{2}+(\mathrm{z} 1-\mathrm{c})^{2}} * 180 / \pi<40 \\ 1-0.5 * \tan ^{-1} \sqrt{(\mathrm{x} 1-\mathrm{a})^{2}+(\mathrm{y} 1-\mathrm{b})^{2}+(\mathrm{z} 1-\mathrm{c})^{2}} * 180 / \pi \\ 40<\tan ^{-1} \sqrt{(\mathrm{x} 1-\mathrm{a})^{2}+(\mathrm{y} 1-\mathrm{b})^{2}+(\mathrm{z} 1-\mathrm{c})^{2}} * 180 / \pi<100 \\ 0, \tan ^{-1} \sqrt{(\mathrm{x} 1-\mathrm{a})^{2}+(\mathrm{y} 1-\mathrm{b})^{2}+(\mathrm{z} 1-\mathrm{c})^{2}} * 180 / \pi>100\end{array}\right.$

The graph of this function is shown in Fig. 1 .
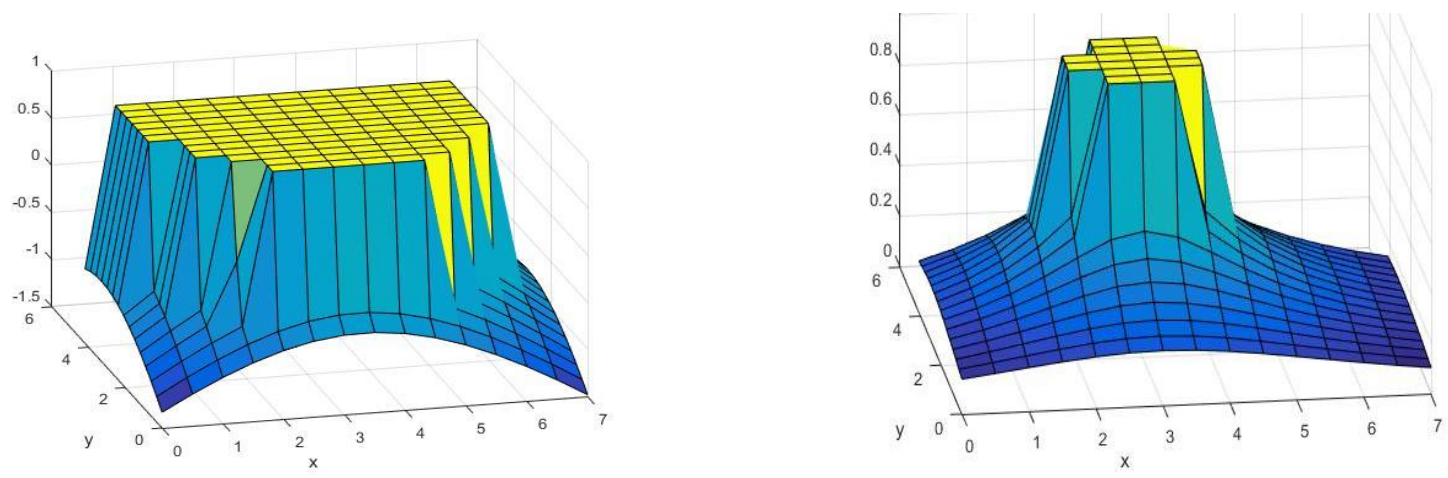

Figure 1.

Finite the

distance

scoring

function

Figure 2.

Finite the angle

scoring

function

The Angle Scoring Function. On the basis of the sensor characteristics, the sensing range is 
only within 100 degrees, and the most sensitive range is within 40 degrees. Therefore, we create the following scoring function:

$$
\left\{\begin{array}{c}
1, \tan ^{-1} \sqrt{(\mathrm{x} 1-\mathrm{a})^{2}+(\mathrm{y} 1-\mathrm{b})^{2}+(\mathrm{z} 1-\mathrm{c})^{2}} * 180 / \pi<40 \\
1-0.5 * \tan ^{-1} \sqrt{(\mathrm{x} 1-\mathrm{a})^{2}+(\mathrm{y} 1-\mathrm{b})^{2}+(\mathrm{z} 1-\mathrm{c})^{2}} * 180 / \pi, \\
40<\tan ^{-1} \sqrt{(\mathrm{x} 1-\mathrm{a})^{2}+(\mathrm{y} 1-\mathrm{b})^{2}+(\mathrm{z} 1-\mathrm{c})^{2}} * 180 / \pi<100 \\
0, \tan ^{-1} \sqrt{(\mathrm{x} 1-\mathrm{a})^{2}+(\mathrm{y} 1-\mathrm{b})^{2}+(\mathrm{z} 1-\mathrm{c})^{2}} * 180 / \pi>100
\end{array}\right.
$$

The graph of above function is shown in Fig. 2.

The Judgment of Shaded Areas. Because the dormitory beds are fixed, we can take a point (x1, $y 1, z 1)$ on the ceiling as an example. First, connect this point with each point of the upper berth, getting a cluster of straight lines. Each line has an intersection point with lower berth, and connecting all the intersection point can get a curve. The curve that is on the side of the wall forms the shadow areas.

The coordinates of the calculated intersection points are shown in the Table 1:

Table 1 The intersection point

\begin{tabular}{|c|r|r|l|l|l|}
\hline $\mathrm{x} 1$ & $\mathrm{y} 1$ & $\mathrm{x} 2$ & $\mathrm{y} 2$ & $\mathrm{x} 3$ & $\mathrm{y} 3$ \\
\hline-0.06451 & -2.77778 & -0.06451 & 6.434568 & 5.958951 & 6.434568 \\
\hline-0.06451 & -2.60062 & -0.06451 & 6.611728 & 5.958951 & 6.611728 \\
\hline-0.06451 & -2.42346 & -0.06451 & 6.788889 & 5.958951 & 6.788889 \\
\hline-0.06451 & -2.2463 & -0.06451 & 6.966049 & 5.958951 & 6.966049 \\
\hline-0.06451 & -2.06914 & -0.06451 & 7.14321 & 5.958951 & 7.14321 \\
\hline-0.06451 & -1.89198 & -0.06451 & 7.32037 & 5.958951 & 7.32037 \\
\hline-0.06451 & -1.71481 & -0.06451 & 7.497531 & 5.958951 & 7.497531 \\
\hline-0.06451 & -1.53765 & -0.06451 & 7.674691 & 5.958951 & 7.674691 \\
\hline-0.06451 & -1.36049 & -0.06451 & 7.851852 & 5.958951 & 7.851852 \\
\hline-0.06451 & -1.18333 & -0.06451 & 8.029012 & 5.958951 & 8.029012 \\
\hline-0.06451 & -1.00617 & -0.06451 & 8.206173 & 5.958951 & 8.206173 \\
\hline-0.06451 & -0.82901 & -0.06451 & 8.383333 & 5.958951 & 8.383333 \\
\hline-0.06451 & -0.65185 & -0.06451 & 8.560494 & 5.958951 & 8.560494 \\
\hline-0.06451 & -0.47469 & -0.06451 & 8.737654 & 5.958951 & 8.737654 \\
\hline-0.06451 & -0.29753 & -0.06451 & 8.914815 & 5.958951 & 8.914815 \\
\hline-0.06451 & -0.12037 & -0.06451 & 9.091975 & 5.958951 & 9.091975 \\
\hline-0.06451 & 0.05679 & -0.06451 & 9.269136 & 5.958951 & 9.269136 \\
\hline
\end{tabular}

In Table 1, x1 and y1 represent the coordinates of the first bed board, $\mathrm{x} 2$ and $\mathrm{y} 2$ represent the coordinates of the second bed board, and $\mathrm{x} 3$ and $\mathrm{y} 3$ represent the coordinates of the third bed. Because all the points are located in the celling, their $\mathrm{z}$ coordinate are $3.3 \mathrm{~m}$.

At the same time, the shadow projected by the sensor on the ceiling is different from the shadow projected by the side wall of the room in shape and volume. The specific difference is shown in the Fig。 3 and Fig. 4 below. of the room on the ceiling 


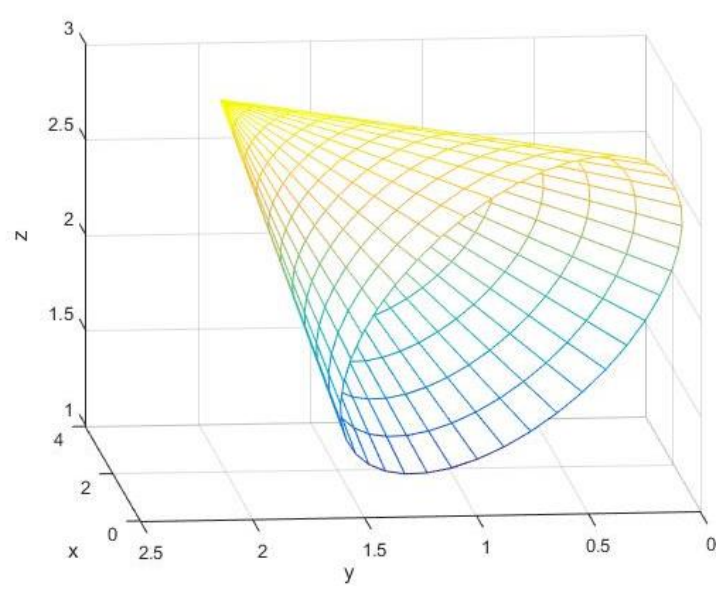

Figure 3. Finite the shadow projected by the side wall

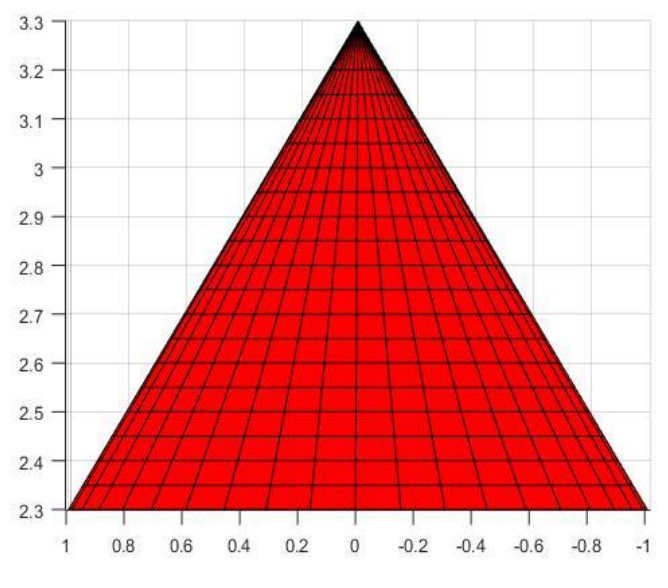

Figure 4. Finite the shadow projected by the sensor

Thus, if we synthetically use the sensor at the ceiling and the sensor at the side wall, we can obtain the best detecting effect. What's more, due to the uses of multiple sensors, several signals will be transmitted to the microcontroller, and after a series of logic operations, the microcontroller output the final detection result. And this process greatly improves the detection accuracy.

In summary, after preliminary analysis and the operations in matlab, we finally determined the number of pyroelectric infrared sensors is three, and one of which is installed on the ceiling, the other two should be installed on the side wall.

\section{The Evaluation of the Model}

1. This model turns the optimal problem into a scoring function, which not only makes selecting standard more distinct, but also lets the results more convincing.

2. This model takes the sensor detection distance and angle into consideration, making the model consistent with the actual situation.

3. In order to distinguish the sensing area and undetectable area of the sensors, our model divides dormitory space into lots of cells, which simplifies the calculation process.

4. The model only considers large shelter, such as bed, cabinet, but not consider other measurement error which caused by other shelter.

\section{Reference}

[1] Zhao Juan. Evaluation Model for Student Dormitory Design Scheme [J]. Journal of Anhui Science and Technology University, 2011, 25(6):55-60.

[2] Peng Lijing, Gong Shikuan, Li Lihong. The principle and application of pyroelectric infrared sensor [J]. Coal Technology, 2005, (03): 113-114.

[3] Yang Bo, Chen Youxian. The principle and application of pyroelectric infrared sensor [J]. Instrument Technique, 2008 (6): 66-68.

[4] Lv Honglin. A talking about the Change of Space Design of Student Dormitory in Colleges and Universities Today [J]. Science and Technology, 2010 (15): 20-21

[5] Zhang Ye, Yang Wei, Yue Yuan, et al. A study on influencing factors of detection distance of pyroelectric infrared sensor [J]. Journal of Infrared and Millimeter Waves, 2010, 29 (6): 448-451.

[6] Luo Xiaomu, Gao Liwen, Luo Xiaoyan, et al. Indoor human body positioning device and its implementation method based on pyroelectric infrared sensor: CN105547494A [P]. 2016.

[7] Huang Qiongxiang, Nasierjiang. Taurson. The sum and analysis of Matlab drawing function [J]. Higher Science Education, 2005 (6): 40-42.

[8] Liu Haoqian. Unmanned monitoring and lighting control device in classroom [J] Electronic Technology and Software Engineering, 2013 (21): 261-262. 
[9] Wang Guoyan. Student dormitory electrical appliances and management countermeasures [J]. Management observation, 2014 (15): 140-141.

[10] Shui Yong. Li Xiaobing. Intelligent Power Management Solution in Dormitory [C]// Sichuan Electric Joint Annual Meeting. 